\title{
Impacto de las apps móviles en la actividad física: un meta-análisis Impact of mobile apps on physical activity: Ameta-analysis
}

\author{
Inmaculada Aznar Díaz, María Pilar Cáceres Reche, Juan Manuel Trujillo Torres, José María Romero Rodríguez
}

Universidad de Granada (España)

\begin{abstract}
Resumen. El uso de aplicaciones (apps) móviles en la práctica deportiva se ha convertido en algo habitual. Cada vez es más común ver a personas practicando deporte mientras utiliza su dispositivo móvil para medir su rendimiento o simplemente por el hecho de estar usando una app lúdica que requiere el desplazamiento. Por tanto, debido a la relevancia de la temática, en este estudio se propuso como objetivo analizar el efecto de las aplicaciones móviles en la actividad física a partir de la revisión de las investigaciones indexadas en las bases de datos Scopus y PubMed (2013-2018). La metodología utilizada ha sido una revisión sistemática con meta-análisis, poniendo el foco de interés concretamente en cinco variables de análisis en base a estudios previos: muestra, aplicación móvil, diseño metodológico, instrumentos de recogida de datos y principales hallazgos. La muestra se compuso por investigaciones de carácter empírico con mínimo de un grupo experimental y otro control (n = 18). Entre los resultados, se constata la variabilidad de apps utilizadas en la actividad física, así como el efecto estadísticamente significativo a favor del grupo experimental. Finalmente, los dispositivos móviles son un potente recurso para la mejora y aumento de la práctica deportiva, al mismo tiempo que se establecen nuevos componentes motivacionales para realizar deporte y sus implicaciones en la enseñanza de la educación física.
\end{abstract}

Palabras clave: Aplicaciones móviles, actividad física, meta-análisis, dispositivos móviles, educación física.

Abstract. The use of mobile applications (apps) in sports has become common practice. It is increasingly common to see people practicing sports while using their mobile device to measure their performance or simply because they are using a playful app that requires movement. Therefore, due to the relevance of the subject, this study aimed to analyse the effect of mobile applications on physical activity from the review of the research indexed in the Scopus and PubMed databases (2013-2018). The methodology used was a systematic review with meta-analysis, placing the focus of interest specifically on five analysis variables based on previous studies: sample, mobile application, methodological design, data collection instruments, and main findings. The sample was composed of empirical research papers with at least one experimental group and another control $(n=18)$. Among the results, the variability of apps used in physical activity is verified, as well as the statistically significant effect in favour of the experimental group. Finally, mobile devices are a powerful resource for the improvement and increase of sports practice, while establishing new motivational components for sports and their implications in the teaching of physical education.

Keywords: Mobile apps, physical activity, meta-analysis, mobile devices, physical education.

\section{Introducción}

La implementación de la tecnología móvil en la actividad física ha iniciado un cambio en el modo y motivaciones para hacer deporte. El uso de dispositivos móviles abre un gran abanico de posibilidades y una infinidad de recursos para mejorar la experiencia de los usuarios. Este hecho se incrementa en materias como la Educación Física, donde existen diversas aplicaciones móviles (apps) que favorecen el aprendizaje de diversos conceptos y facilitan la dinámica de trabajo en el aula (Filgueira, 2016).

Concretamente, el uso de dispositivos móviles en la enseñanza se denomina aprendizaje móvil o mobile learning. Esta metodología docente se define por aplicar los dispositivos móviles para mediar en el proceso de enseñanza-aprendizaje(Aznar, Romero \& Rodríguez-García, 2018).

Algunos de los principales beneficios del uso de la tecnología en el desarrollo humano, se encuentran en el ahorro de tiempo, la facilidad de acceso a la información y sobre todo a la mejora en la realización de diversas tareas cotidianas (Gómez, Trujillo, Aznar \& Cáceres, 2018). En cambio, la adicción y los problemas de salud generados por el uso abusivo de la tecnología son contrapartidas que hay que tener en cuenta y tomar medidas para su prevención (García-Umaña \& Tirado, 2018).

Por su parte, el uso de los dispositivos móviles para realizar cualquier tipo de actividad física es algo reciente, teniendo en cuenta que la aplicación de las Tecnologías de la Información y Comunicación (TIC) presentan un corto recorrido en esteámbito (Prat, Camerino \& Coiduras, 2013; Rodríguez-García, Romero \& Agreda, 2019). Aun así, nos encontramos con varias experiencias de implementación de los dispositivos móviles en la Educación Física: Monguillot, González, Guitert y Zurita (2014) combinan el mobile learning a través de los códigos QR para el fomento de prácticas físicas saludables; Nagovitsyn, Vladykina y Senator (2015) desarrollan fundamentos metodológicos y tecnológicos de aprendizaje móvil para facilitar la promoción y mejora de la actividad motriz

Fecha recepción: 08-07-18. Fecha de aceptación: 05-01-19 José María Romero Rodríguez romejo@ugr.es de los jóvenes; Castro y Gómez (2016) utilizan en la etapa de educación secundaria los códigos QR, obteniendo como resultados una mejora en la actitud y motivación de los estudiantes; Marcelo, Yot, Álvarez, Ortega y Aros (2016) implementan una app que detecta la ubicación, cantidad de tiempo y la actividad física realizada.

Asimismo, las apps son uno de los principales recursos vinculados directamente con el uso de dispositivos móviles. Filgueira (2014) realiza una tipología en función de su uso para la práctica deportiva, entrándonos con una amplia variedad: aplicaciones de medición de la actividad física; adquisición de hábitos; fitness; deportes específicos; trabajo en equipo y; de carácter lúdico. En consonancia, se resaltan una serie de ventajas asociadas al uso de los dispositivos móviles en la actividad física: aumento de la motivación, facilidad para recabar información, ubicuidad, integración de usuarios con lesiones y reducción de costes (García \& Sánchez, 2014; Quintero, Jiménez \& Area, 2018). En cambio, las principales limitaciones se centran en la competencia digital del profesorado y las restricciones y/o falta de recursos en etapas educativas como la educación primaria y secundaria (Díaz, 2018). En este sentido, para que su implementación sea efectiva en todos los contextos educativos, es necesario el establecimiento de políticas comunes en el centro y la estrategia BYOD, que hace referencia al hecho de que cada estudiante traiga su propio dispositivo al centro (Suárez, Lloret \& Mengual, 2016).

Por otro lado, diversas investigaciones empiezan a mostrar resultados positivos en cuanto al aumento de la actividad física a partir del uso de dispositivos tecnológicos y/o apps (Fanning, Mullen \& McAuley, 2012; Conroy, Yang \& Maher, 2014; Muntaner, Vidal-Conti \& Palou, 2016; Leirós, Arce, García-Soidán \& Naveira, 2017; Marqués, Cela \& Gisbert, 2017; Pérez-López, Rivera \& Trigueros, 2017; Guillen, Herrera \& Ale, 2018). Lo cual conlleva, entre otros, el desarrollo de la competencia digital de los usuarios (Monguillot et al., 2014; Gallego, Muñoz, Arribas \& Rubia, 2016). Sin embargo, otros estudios recogen datos totalmente contrarios que establecen efectos negativos en su uso, e incluso la inexistencia de diferencias estadísticamente significativas entre grupos que utilizan apps y los que siguen una rutina deportiva sin recursos electrónicos (Greco, Tambolini, Ambruosi \& Fischetti, 2017; Fukuoka et al., 2018). No obstante, cabe indicar, que el peso corporal es otra constante que se ve afectada por la actividad física (Cuadri, Torne- 
ro, Sierra \& Sáez, 2018; Martínez-López, Moreno, Suarez \& RuizAriza, 2018; Lozano, Zurita, Ubago, Puertas, Ramírez \& Núñez, 2019; Núñez, Zurita, Ramírez, Lozano, Puertas \& Ubago, 2019). Por lo que es de interés considerar esta variable en la revisión de la literatura y en el propio meta-análisis.

Por tanto, debido a la relevancia de la temática, los estudios contradictorios y el escaso recorrido actual en el uso de dispositivos móviles en la práctica deportiva, el objetivo de este trabajo ha sido analizar el efecto de las aplicaciones móviles en la actividad física a partir de la revisión de las investigaciones indexadas en las bases de datos Scopus y PubMed (2013-2018). Asimismo, las preguntas de investigación que guiaron y estructuraron el trabajo se relacionaron con:

- ¿El ¿ ¿ co de aplicaciones móviles mejora la actividad física?

- ¿Qué tipo de aplicaciones móviles son las más utilizadas para realizar cualquier actividad física?

- ¿ ¿Cuál es el efecto real del uso de las aplicaciones móviles en la salud física de los usuarios?

\section{Metodología}

Debido a las características del estudio, se ha seguido una metodología de revisión sistemática con meta-análisis (Soler, Sánchez-Meca, López \& Navarro, 2014). Además, se han tenido en cuenta los criterios de elegibilidad y de selección de los estudios establecidos en la declaración PRISMA para revisiones sistemáticas y meta-análisis (Urrútia \& Bonfill, 2010).

La búsqueda se ha realizado en las bases de datos Scopus y PubMed, siguiendo las consideraciones de estudios previos en la misma línea (Muntaner, Vidal-Conti \& Palou, 2016). Asimismo, las palabras clave responden en primera instancia a la temática objeto de estudio y fueron extraídas del tesauro ERIC, entre ellas: mobile devices, mobile apps, smartphones y physical activities. Para dotar de rigurosidad a la búsqueda, se cruzaron las palabras clave unificadas con el operador booleano AND. De modo que se establecieron las siguientes ecuaciones de búsqueda: «Mobile apps» AND «Physical activity»; «Mobile devices» AND «Physical activity» y; Smartphones AND «Physical activity».

A partir de los resultados iniciales, se aplicaron una serie de criterios de inclusión y exclusión para reducir y discriminar la producción científica de interés para el estudio (Aznar, Cáceres \& Romero, 2018).

\section{Criterios de inclusión}

1. Artículos de revista.

2. Publicaciones entre enero de 2013 y junio de 2018.

3. Publicado en abierto y disponible para su consulta.

4. Uso de cualquier tipo de aplicación móvil en la realización de una actividad física.

5. Estudios empíricos con diseño experimental o cuasiexperimental.

6. Mínimo de un grupo control y otro experimental.

\section{Criterios de exclusión}

1. Actas de congresos, capítulos de libro, libros u otro tipo de publicaciones.

2. Acceso restringido a la publicación.

3. No se utilizan aplicaciones móviles.

4. Estudios teóricos o revisiones.

5. Artículos duplicados.

\section{Muestra}

En concreto, el proceso de determinación de la muestra final ( $\mathrm{n}$ = 18) se ha dividido en diferentes fases: (i) introducción de las ecuaciones de búsqueda en Scopus y PubMed; (ii) aplicación de los criterios 1, 2, 3 de inclusión y 1, 2 de exclusión; (iii) revisión de títulos y resúmenes en función de los criterios 4, 5, 6 de inclusión y 3, 4, 5 de exclusión (figura 1).

De la muestra resultante, se analizaron cinco variables en base a las aportaciones de revisiones sistemáticas previas (Conde \& Tercedor,

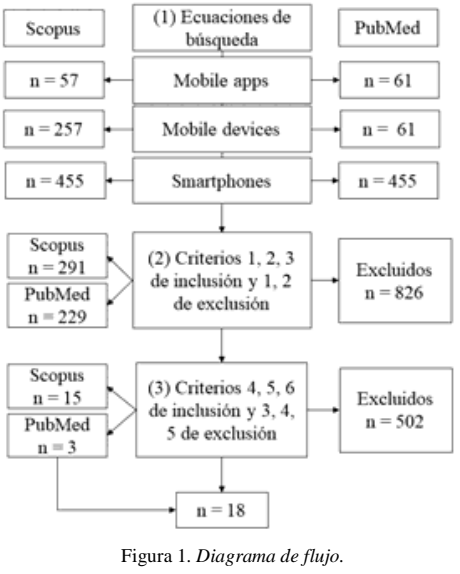

2015; Casanova \& Yuste, 2016; Chacón-Borrego, Ubago, La Guardia, Padial \& Cepero, 2018; Moreno, Fernández, Linares y Espejo, 2018). Estas variables se han clasificado según su tipología (Sánchez-Meca, 2003): sustantivas, muestra objeto de estudio (V1) y aplicación móvil (V2); metodológicas, diseño metodológico(V3) e instrumentos de recogida de datos (V4) y; principales hallazgos (V5).

Por otro lado, el meta-análisis ha permito obtener el tamaño del efecto (TE) global de las distintas investigaciones. Los datos han sido analizados a través del programa Review Manager, versión 5.3.

\section{Resultados}

\section{Variables sustantivas}

La muestra objeto de estudio(V1) de las diferentes investigaciones presenta un tamaño muestral que oscila entre un rango de 42 y 32.000 sujetos $(M=2219$, DT $=7500,15)$. Además, se caracterizan por tener una población diversa: pacientes con diabetes tipo 2 (Alonso et al., 2017; Höchsmann et al., 2017; Valentiner et al., 2017; Bonn et al., 2018); obesidad (Steinberg, Levine, Askew, Foley \& Bennett, 2013; Yang et al., 2017; Turner-McGrievy et al., 2017); adolescentes (Direito, Jiang, Whittaker \& Maddison, 2015); mujeres (Willcox et al., 2017; Fukuoka, Lindgren, Dov, Hooper \& Aswani, 2018; Mascarenhas, Maylin, Vittinghoff, Van \& Hecht, 2018); estudiantes (Gabbiadini \& Greitemeyer, 2018); deportistas (Martine, Mennes, Alpay, Bijwaard \& Baart, 2015) y; población general (Glynn et al., 2013; Van Dantzig, Geleijnse \& Van Halteren, 2013; Safran, Madar \& Shahar, 2015; Althoff, White \& Horvitz, 2016; Howe et al., 2016) (figura 2).

La muestra mayoritaria en los estudios corresponde a una población general (28\%), seguida de pacientes con diabetes tipo 2 (22\%), obesidad (17\%), mujeres (17\%), deportistas (6\%), estudiantes (5\%) y adolescentes (5\%).

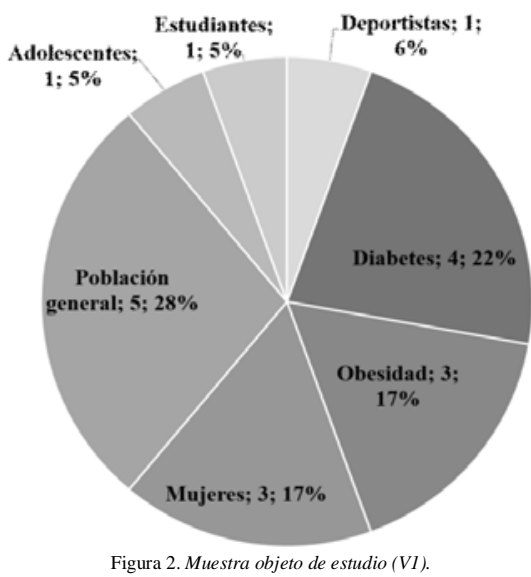

En relación a la aplicación móvil (V2), se han utilizado diferentes apps dependiendo de los objetivos del estudio para comprobar su efecto en la actividad física. Así pues, en pacientes con diabetes tipo 2 se 
investigaron las apps EVIDENT II(Alonso et al., 2017), MOBIGAME (Höchsmann et al., 2017), InterWalk (Valentiner et al., 2017) y DiaCert (Bonn et al., 2018); en personas con obesidad se comprobó el impacto para perder peso y aumentar la actividad física de las apps iMessage (Steinberg et al., 2013), FatSecret (Turner-McGrievy et al., 2017) y HAPPY ME (Yang et al., 2017); en la mejora de la actividad física de adolescentes se implementaron Get Running Couch y Zombies, Run! (Direito et al., 2015); en la población de mujeres que han sido madres se establecieron las apps Nike+ y Sworkit (Mascarenhas et al., 2018); en estudiantes Google Fit, Pacer, Stepz y Pedometer (Gabbiadini \& Greitemeyer, 2018); la muestra de deportistas, referentes a corredores, se utilizaron RunKeeper, Runtastic, Endomundo y Strava (Martine et al., 2015). Por último, en la población general se indagó el efecto de apps comoAccupedo (Glynn et al., 2013), SitCoach(Van Dantzig, Geleijnse \& Van Halteren, 2013), eBalance (Safran, Madar \& Shahar, 2015) y Pokémon Go (Althoff, White \& Horvitz, 2016; Howe et al., 2016). Cabe resaltar que en los estudios de Willcox et al. (2017) y Fukuoka et al. (2018) no se especifica la app empleada.

\section{Variables metodológicas}

El diseño metodológico (V3) más utilizado en los diferentes estudios es el diseño experimental con un grupo control y otro experimental y medidas pretest y postest (68\%) (Glynn et al., 2013; Steinberg et al., 2013; Safran, Madar \& Shahar, 2015; Althoff, White \& Horvitz, 2016; Alonso et al., 2017; Höchsmann et al., 2017; Turner-McGrievy et al., 2017; Valentiner et al., 2017; Willcox et al., 2017; Bonn et al., 2018; Gabbiadini \& Greitemeyer, 2018; Mascarenhas et al., 2018). En minoría, también se aplica el diseño experimental con un grupo control y dos experimentales, pretest y postest (11\%) (Direito et al., 2015; Fukuoka et al., 2018), experimental con comparación entre poblaciones similares y medidas pretest y postest (11\%) (Martine et al., 2015; Howe et al., 2016), experimental con grupo control y otro experimental y solo postest (5\%) (Yang et al., 2017) y cuasiexperimental con grupo control y experimental, pretest y postest (5\%) (Van Dantzig, Geleijnse \& Van Halteren, 2013).

En cuanto a los instrumentos de recogida de datos (V4), se agrupan principalmente en cinco: cuestionario (Martine et al., 2015; Safran, Madar \& Shahar, 2015; Howe et al., 2016; Alonso et al., 2017; Valentiner et al., 2017; Willcox et al., 2017; Yang et al., 2017; Gabbiadini y Greitemeyer, 2018; Mascarenhas et al., 2018); acelerómetro (Direito et al., 2015; Althoff, White \& Horvitz, 2016; Höchsmann et al., 2017; Bonn et al., 2018; Fukuoka et al., 2018); escala (Glynn et al., 2013); test (Van Dantzig, Geleijnse \& Van Halteren, 2013) y; dinamómetro (Steinberg et al., 2013; Turner-McGrievy et al., 2017). En concreto, el cuestionario es el más utilizado para comprobar el efecto de las apps móviles en la actividad física (50\%), después el acelerómetro (28\%), dinamómetro (11\%), test (6\%) y escala (5\%) (figura 3).

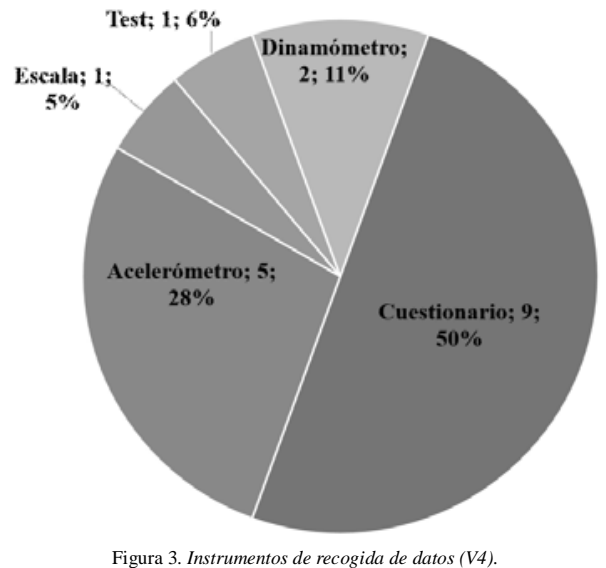

\section{Principales hallazgos}

Entre las investigaciones analizadas, únicamente se han podido recopilar los principales hallazgos (V5) de 11 de ellas, debido a que las siete restantes se tratan de protocolos clínicos y/o diseños de investigaciones a implementar en un futuro (Glynn et al., 2013; Direito et al., 2015; Alonso et al., 2017; Höchsmann et al., 2017; Yang et al., 2017; Valentiner et al., 2017; Bonn et al., 2018). De este modo, nos encontramos con resultados diversos en torno al impacto de las apps móviles en la actividad física: la mensajería de texto puede ser una herramienta útil de autocontrol para el peso (Steinberg et al., 2013); potencial del uso de un smartphone como plataforma para reducir el comportamiento sedentario (Van Dantzig, Geleijnse \& Van Halteren, 2013); el uso de apps móviles es beneficioso en la preparación de una maratón, puesto que promueve la salud y la actividad física (Martine et al., 2015); ); la app motiva a los usuarios a aumentar significativamente la duración semanal de actividad física (Safran, Madar \& Shahar, 2015); Pokémon Go se asocia con un incremento en el número de pasos después de su instalación (Howe et al., 2016); Pokémon Go aumenta significativamente la actividad física en un período de 30 días (Althoff, White \& Horvitz, 2016); mayor pérdida de peso en el grupo experimental (TurnerMcGrievy et al., 2017); actividad física positiva y menor aumento de peso de mujeres en periodo gestacional (Willcox et al., 2017); no existen diferencias en los niveles de actividad física entre grupos (Fukuoka et al., 2018); efecto positivo en el comportamiento saludable al utilizar una fitness app (Gabbiadini \& Greitemeyer, 2018) y; la intervención aumentó la actividad física en madres inactivas (Mascarenhas et al., 2018).

A modo de resumen se recogen las cinco variables analizadas en una única tabla (tabla 1$)$.

\begin{tabular}{|c|c|c|c|c|c|}
\hline Estudio & Muestra & App & Diseño & istrumento & Iallazgos \\
\hline $\begin{array}{l}\text { Alonso et al. } \\
\text { (2017) }\end{array}$ & $\begin{array}{l}\mathrm{n}=200 \\
\text { diabetes }\end{array}$ & $\begin{array}{c}\text { EVIDENT } \\
\text { II }\end{array}$ & $\begin{array}{l}\text { Experimental } \\
\text { GC y GE } \\
\text { pretest y postest }\end{array}$ & Cuestionario & -- \\
\hline $\begin{array}{l}\text { Althoff, } \\
\text { White y } \\
\text { Horvitz } \\
(2016) \\
\end{array}$ & $\begin{array}{c}\mathrm{n}=32.000 \\
\text { población } \\
\text { general }\end{array}$ & $\begin{array}{l}\text { Pokémon } \\
\text { Go }\end{array}$ & $\begin{array}{l}\text { Experimental } \\
\text { GC y GE } \\
\text { pretest y postest }\end{array}$ & Acelerómetro & $\begin{array}{l}\text { Aumento de la } \\
\text { actividad física } \\
\text { en } 30 \text { días }\end{array}$ \\
\hline $\begin{array}{l}\text { Bonn et al. } \\
\text { (2018) }\end{array}$ & $\begin{array}{l}\mathrm{n}=250 \\
\text { diabetes }\end{array}$ & DiaCert & $\begin{array}{c}\text { Experimental } \\
\text { GC y GE } \\
\text { pretest y postest }\end{array}$ & Acelerómetro & -- \\
\hline $\begin{array}{l}\text { Direito et al. } \\
\text { (2015) }\end{array}$ & $\begin{array}{l}\mathrm{n}=51 \\
\text { adolescentes }\end{array}$ & $\begin{array}{c}\text { Get } \\
\text { Running y } \\
\text { Zombies, } \\
\text { Run! } \\
\end{array}$ & $\begin{array}{c}\text { Experimental } \\
\text { GC y } 2 \text { GE } \\
\text { pretest y postest }\end{array}$ & Acelerómetro & -- \\
\hline $\begin{array}{l}\text { Fukuoka et } \\
\text { al. (2018) }\end{array}$ & $\begin{array}{l}\mathrm{N}=203 \\
\text { mujeres }\end{array}$ & -- & $\begin{array}{c}\text { Experimental } \\
\text { GC y } 2 \text { GE } \\
\text { pretest y postest }\end{array}$ & Acelerómetro & $\begin{array}{l}\text { No diferencias } \\
\text { entre grupos }\end{array}$ \\
\hline $\begin{array}{l}\text { Gabbiadini y } \\
\text { Greitemeyer } \\
\quad(2018)\end{array}$ & $\begin{array}{l}\quad \mathrm{n}=78 \\
\text { estudiantes }\end{array}$ & $\begin{array}{c}\text { Google Fit, } \\
\text { Pacer, } \\
\text { Stepz y } \\
\text { Pedometer }\end{array}$ & $\begin{array}{l}\text { Experimental } \\
\text { GC y GE } \\
\text { pretest y postest }\end{array}$ & Cuestionario & $\begin{array}{c}\text { Efecto positivo } \\
\text { en el } \\
\text { comportamiento } \\
\text { saludable }\end{array}$ \\
\hline $\begin{array}{l}\text { Glynn et al. } \\
\text { (2013) }\end{array}$ & $\begin{array}{c}\mathrm{n}=80 \\
\text { población } \\
\text { general }\end{array}$ & Accupedo & $\begin{array}{c}\text { Experimental } \\
\text { GC y GE } \\
\text { pretest y postest }\end{array}$ & Escala & -- \\
\hline $\begin{array}{l}\text { Höchsmann } \\
\text { et al. (2017) }\end{array}$ & $\begin{array}{l}\mathrm{n}=42 \\
\text { diabetes }\end{array}$ & Mobigame & $\begin{array}{c}\text { Experimental } \\
\text { GC y GE } \\
\text { pretest y postest }\end{array}$ & Acelerómetro & -- \\
\hline $\begin{array}{l}\text { Howe et al. } \\
\text { (2016) }\end{array}$ & $\begin{array}{c}\mathrm{n}=1182 \\
\text { población } \\
\text { general }\end{array}$ & $\begin{array}{l}\text { Pokémon } \\
\text { Go }\end{array}$ & $\begin{array}{l}\text { Experimental } \\
\text { poblaciones } \\
\text { similares } \\
\text { pretest y postest }\end{array}$ & Cuestionario & $\begin{array}{c}\text { Incremento en el } \\
\text { número de pasos } \\
\text { después de su } \\
\text { instalación }\end{array}$ \\
\hline $\begin{array}{l}\text { Martine et al. } \\
\text { (2015) }\end{array}$ & $\begin{array}{c}\mathrm{n}=4307 \\
\text { deportistas }\end{array}$ & $\begin{array}{c}\text { RunKeeper } \\
\text {, Runtastic, } \\
\text { Endomund } \\
\text { o y Strava }\end{array}$ & $\begin{array}{l}\text { Experimental } \\
\text { poblaciones } \\
\text { similares } \\
\text { pretest y postest }\end{array}$ & Cuestionario & $\begin{array}{c}\text { Las apps } \\
\text { promueven la } \\
\text { salud y la } \\
\text { actividad física }\end{array}$ \\
\hline $\begin{array}{l}\text { Mascarenhas } \\
\text { et al. (2018) }\end{array}$ & $\begin{array}{l}\mathrm{n}=64 \\
\text { mujeres }\end{array}$ & $\begin{array}{l}\text { Nike+ y } \\
\text { Sworkit }\end{array}$ & $\begin{array}{l}\text { Experimental } \\
\text { GC y GE } \\
\text { pretest y postest }\end{array}$ & Cuestionario & $\begin{array}{l}\text { Aumento de la } \\
\text { actividad física } \\
\text { en madres } \\
\text { inactivas }\end{array}$ \\
\hline $\begin{array}{l}\text { Safran, } \\
\text { Madar y } \\
\text { Shahar } \\
(2015)\end{array}$ & $\begin{array}{l}\mathrm{n}=85 \\
\text { población } \\
\text { general }\end{array}$ & eBalance & $\begin{array}{l}\text { Experimental } \\
\text { GC y GE } \\
\text { pretest y postest }\end{array}$ & Cuestionario & $\begin{array}{c}\text { Aumento } \\
\text { significativo de } \\
\text { la duración } \\
\text { semanal de } \\
\text { actividad física }\end{array}$ \\
\hline $\begin{array}{l}\text { Steinberg et } \\
\text { al. (2013) }\end{array}$ & $\begin{array}{c}\mathrm{n}=50 \\
\text { obesidad }\end{array}$ & iMessage & $\begin{array}{c}\text { Experimental } \\
\text { GC y GE } \\
\text { pretest y postest }\end{array}$ & Dinamómetro & $\begin{array}{c}\text { Herramienta útil } \\
\text { de autocontrol de } \\
\text { peso }\end{array}$ \\
\hline $\begin{array}{c}\text { Turner- } \\
\text { McGrievy et } \\
\text { al. (2017) }\end{array}$ & $\begin{array}{c}\mathrm{n}=81 \\
\text { obesidad }\end{array}$ & FatSecret & $\begin{array}{c}\text { Experimental } \\
\text { GC y GE } \\
\text { pretest y postest }\end{array}$ & Dinamómetro & $\begin{array}{l}\text { Mayor pérdida } \\
\text { de peso en el GE }\end{array}$ \\
\hline $\begin{array}{l}\text { Valentiner et } \\
\text { al. (2017) }\end{array}$ & $\begin{array}{l}\mathrm{n}=246 \\
\text { diabetes }\end{array}$ & InterWalk & $\begin{array}{l}\text { Experimental } \\
\text { GC y GE } \\
\text { pretest y postest }\end{array}$ & Cuestionarios & -- \\
\hline $\begin{array}{c}\text { Van Dantzig, } \\
\text { Geleijnse y } \\
\text { Van Halteren } \\
(2013) \\
\end{array}$ & $\begin{array}{c}\mathrm{n}=86 \\
\text { población } \\
\text { general }\end{array}$ & SitCoach & $\begin{array}{c}\text { Cuasiexperimen } \\
\text { tal con GC y } \\
\text { GE pretest y } \\
\text { postest }\end{array}$ & Test & $\begin{array}{c}\text { Potencial del } \\
\text { smartphone para } \\
\text { reducir el } \\
\text { sedentarismo } \\
\end{array}$ \\
\hline $\begin{array}{l}\text { Willcox et al. } \\
\text { (2017) }\end{array}$ & $\begin{array}{l}\mathrm{n}=91 \\
\text { mujeres }\end{array}$ & -- & $\begin{array}{c}\text { Experimental } \\
\text { GC y GE } \\
\text { pretest y postest }\end{array}$ & Cuestionario & $\begin{array}{c}\text { Actividad física } \\
\text { positiva y menor } \\
\text { aumento de peso }\end{array}$ \\
\hline $\begin{array}{c}\text { Yang et al. } \\
\text { (2017) }\end{array}$ & $\begin{array}{l}\mathrm{n}=846 \\
\text { obesidad }\end{array}$ & Happy Me & $\begin{array}{c}\text { Experimental } \\
\text { GC y GE } \\
\text { postest }\end{array}$ & Cuestionario & -- \\
\hline
\end{tabular}




\section{Meta-análisis}

De la muestra final $(\mathrm{n}=18)$, solo se ha podido realizar el metaanálisis en nueve de ellas (Steinberg et al., 2013; Van Dantzig, Geleijnse \& Van Halteren, 2013; Safran, Madar \& Shahar, 2015; Howe et al., 2016; Turner-McGrievy et al., 2017; Willcox et al., 2017; Fukuoka et al., 2018; Gabbiadini \& Greitemeyer, 2018). Se han excluido los estudios de protocolos clínicos y/o diseños de investigaciones $(n=7)$ y aquellos artículos en los que faltaba información clave para medir el tamaño del efecto ( $n=2$ ). No obstante, en Fukuoka et al. (2018) se han incluido los dos grupos experimentales, así como su efecto en diferentes pruebas de actividad física: (a), (b) corresponden a caminar y (c), (d) a caminar rápido.

Se ha optado por realizar dos meta-análisis debido a que los estudios miden distintos tipos de variables para constatar el efecto en la actividad física: la propia actividad física y el peso corporal.

Si atendemos a los datos del meta-análisis de la variable actividad física, se observan diferencias estadísticamente significativas ( $\mathrm{p}=.008$ ). El diagrama de bosque confirma la significación a favor del grupo experimental, puesto que la figura del diamante se posiciona en el lado derecho (figura 4). De modo que se verifica el aumento de la actividad física a partir del uso de apps móviles, especificado en el TE global de las investigaciones. En suma, los trabajos de Van Dantzig, Geleijnse y Van Halteren (2013), Safran, Madar y Shahar (2015) y Gabbiadini y Greitemeyer (2018) muestran un efecto positivo en el tratamiento. Mientras que los estudios que rozan la línea central (línea de no efecto) recogen la inexistencia de diferencias entre grupos, situándose Howe et al. (2016) y Fukuoka et al. (2018).

Por otro lado, el meta-análisis de la variable peso detalla un TE no significativo $(p=.09)$. En el diagrama de bosque se observa que el diamante roza la línea de no efecto, por lo que no es significativo en su conjunto a pesar de su posicionamiento en el extremo a favor del grupo experimental (figura 5). En concreto, los estudios de Turner-McGrievy et al. (2017) y Willcox et al. (2017) tienen un efecto positivo a favor del grupo experimental y Steinberg et al. (2013) no presenta efecto entre grupos.

\section{Discusión}

Los principales hallazgos obtenidos se relacionan en primera instancia con la premisa de que el uso de apps mejora la actividad física e incide en la disminución del peso corporal. A este respecto, el proceso sistemático seguido ha permitido realizar una revisión sistemática acorde a los criterios de elegibilidad y de selección de los estudios de la declaración PRISMA(Urrútia \& Bonfill, 2010). Además, las variables analizadas siguen patrones similares a los factores de interés de distintos estudios previos (Conde \& Tercedor, 2015; Casanova \& Yuste, 2016; Chacón-Borrego et al., 2018; Moreno et al., 2018).

Atendiendo a la tipología de las apps utilizadas (Filgueira, 2014), la mayoría de ellas se relacionan con aplicaciones de medición de la actividad física, aunque también se encuentran de tipo adquisición de hábitos y lúdicas. En este sentido, las apps móviles de carácter lúdico se posibilitan como un recurso de interés para el aumento de la actividad física, las cuales llevan asociado un componente motivacional intrínseco. Esto es, una pieza clave para reforzar la actividad física a través del juego, con apps como Pokémon Go o Zombies Run!

En esta línea, la mayor parte de los estudios refleja las ventajas asociadas al uso de apps para la actividad física, fijando el foco especialmente en el aumento de la motivación y laubicuidad (García \& Sánchez, 2014; Quintero, Jiménez \& Area, 2018).

Algunos de los estudios han puesto el interés en el aumento de la actividad física en la población de pacientes con diabetes tipo 2 y en la población de personas con obesidad, subrayando una serie de beneficios y obteniendo resultados satisfactorios (Turner-McGrievy et al., 2017). Esta constante, abre un abanico de posibilidades para hacer frente a diversas enfermedades a partir del uso de los dispositivos móviles.

En consonancia, no es de extrañar que los recursos tecnológicos empiecen a implementarse en la educación física para mejorar el aprendizaje de la materia (Filgueira, 2016). Este escenario de expansión de los dispositivos móviles en el ámbito educativo acontece grandes cambios en la enseñanza tradicional de la educación física. Asimismo, los recursos emergentes como la realidad aumentada y la realidad virtual se alzan como potentes herramientas en la educación del siglo XXI (Aznar, Romero \& Rodríguez-García, 2018). Un reflejo de ello, es la cantidad de apps móviles que se utilizan para favorecer la actividad física y que quedan recogidas en este estudio de revisión sistemática con metaanálisis.

Centrándonos en el meta-análisis, se constata que el tamaño del efecto global de las investigaciones es estadísticamente significativo a favor del grupo experimental. Por lo que se evidencia un efecto positivo de las apps móviles en la actividad física, en la misma línea que las consideraciones de diversos autores (Fanning, Mullen \& McAuley, 2012; Conroy, Yang \& Maher, 2014; Muntaner, Vidal-Conti \& Palou, 2016; Leirós et al., 2017; Marqués, Cela \& Gisbert, 2017; Guillen, Herrera \& Ale, 2018). Por el contrario, no se han encontrado efectos negativos del uso de las apps en la actividad física como remarca Greco et al. (2017). Únicamente, si se evidencia en algunos de ellos el efecto inexistente entre grupos control y experimental.

En suma, el uso de apps móviles tiene un impacto positivo en la actividad física que no solo se limita a un aumento en los niveles de

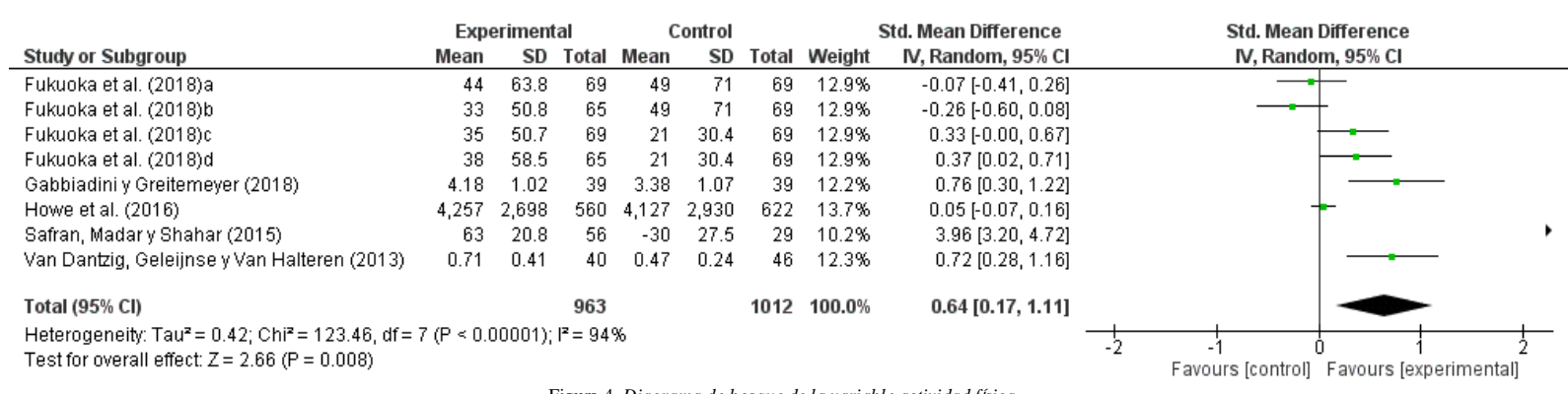

Figura 4. Diagrama de bosque de la variable actividad física.

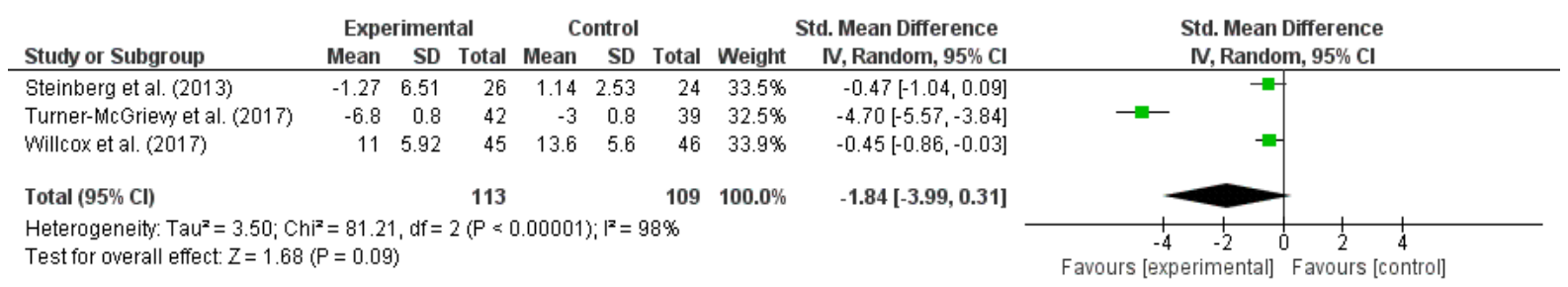

Figura 5. Diagrama de bosque de la variable peso corporal. 
actividad deportiva, sino que lleva aparejados ciertos beneficios como un aumento de la motivación y el posible desarrollo de competencias digitales (Monguillot et al., 2014; Gallego et al., 2016).

No obstante, las principales limitaciones del estudio se relacionan con el número de investigaciones que tienen como objeto de estudio las apps móviles, el cual es reducido. Este hecho puede deberse a las premisas remarcadas por Prat, Camerino y Coiduras (2013) acerca del escaso recorrido de las TIC en la educación física. Además, la especialización en las bases de datos consultadas (Scopus y PubMed) ha hecho que se prescinda de otros artículos que se encuentren fuera de ellas. Sin embargo, creemos pertinente consultar en un primer momento los artículos indexados en estas bases científicas. A su vez, aquellos estudios que no recogían los datos referentes a la media y desviación típica se han suprimido, puesto que no aportan la información necesaria para la realización de un meta-análisis.

Finalmente, como futuras líneas de investigación derivadas de este estudio, se establece la posibilidad de seguir indagando sobre el efecto de las apps móviles en la actividad física. También, sería de interés comenzar a investigar el impacto de los dispositivos móviles y de la metodología mobile learning en la educación física, concretamente en las etapas educativas de Educación Primaria y Secundaria.

\section{Conclusiones}

El presente estudio sigue la línea de investigación sobre la implementación de apps móviles en la práctica deportiva, remarcando ciertos beneficios en su uso. El ritmo frenético de la tecnología está impactando en nuestras vidas de forma considerable, por ello se debe adaptar cuidadosamente su uso para sacar todo el potencial que nos ofrecen. Sin olvidar, que una mala utilización puede conllevar a una serie de prejuicios para nuestra salud.

Así pues, el mercado creciente de apps móviles se encuentra en una etapa incipiente, donde existe una gran oferta de apps para la mejora de la actividad física. El reto que se nos avecina será el de discernir entre una app que realmente tenga un efecto consistente de una que no favorezca la actividad física.

Por el momento, danto respuesta a los interrogantes planteados en este trabajo, queda claro que el uso de ciertas apps mejora la actividad física como recogen diversos estudios (Van Dantzig, Geleijnse \& Van Halteren, 2013; Safran, Madar \& Shahar, 2015; Gabbiadini \& Greitemeyer, 2018). También se constata que el tipo de apps móviles más utilizadas son las de medición de la actividad física, entre ellas RunKeeper, Runtastic y eBalance. En suma, respondiendo a la última cuestión acerca de ¿cuál es el efecto real del uso de las aplicaciones móviles en la salud física de los usuarios?, podemos afirmar que es un efecto positivo a favor de la mejora de la actividad física y la pérdida de peso corporal, lo cual repercute en la salud de los usuarios.

En consecuencia, con este trabajo se ha dado respuesta al objetivo planteado acerca de analizar el efecto de las aplicaciones móviles en la actividad física a partir de la revisión de las investigaciones indexadas en las bases de datos Scopus y PubMed (2013-2018).

Por último, se remarcan diversas implicaciones para la comunidad científica a raíz de la confección del meta-análisis:

- Actualmente el cómputo de trabajos sobre la temática es escaso, es necesario seguir investigando sobre el efecto de las apps móviles en la actividad física y sobre todo en la educación física.

- Los dispositivos móviles son un potente recurso para la mejora y aumento de la práctica deportiva.

- Las apps móviles de carácter lúdico establecen nuevos componentes motivacionales para realizar deporte, de modo que el deseo de mejorar el estado físico es una consecuencia secundaria de conseguir los logros establecidos en el juego.

- El uso de apps para la actividad física favorece el tratamiento de enfermedades como la diabetes tipo 2 y la obesidad.

- Los datos obtenidos del tamaño del efecto global del metaanálisis de los distintos estudios es significativo, con efecto positivo en el grupo experimental.

\section{Referencias}

Alonso, R., Gómez, M.A., Patino, M.C., Sánchez, N., Agudo, C., Castaño, C., García, L., \& Recio, J.I. (2017). Effectiveness of a multifactorial intervention based on an application for smartphones, heart-healthy walks and a nutritional workshop in patients with type 2 diabetes mellitus in primary care (EMID): study protocol for a randomised controlled trial. BMJ Open, 7, 1-8. DOI:10.1136/bmjopen-2017016191

Althoff, T., White, R., \& Horvitz, E. (2016). Influence of Pokémon Go on Physical Activity: Study and Implications. Journal of Medical Internet Research, 18(12), 1-14. DOI:10.2196/jmir.6759

Aznar, I., Cáceres, M.P., \& Romero, J.M. (2018). Efecto de la metodología mobile learning en la enseñanza universitaria: meta-análisis de las investigaciones publicadas en WOS y Scopus. RISTI. Revista Ibérica de Sistemas y Tecnologías de la Información, 30, 1-16.

Aznar, I., Romero, J.M., \& Rodríguez-García, A.M. (2018). La tecnología móvil de Realidad Virtual en educación: una revisión del estado de la literatura científica en España. EDMETIC, Revista de Educación Mediática y TIC, 7(1), 256-274. DOI:10.21071/edmetic.v7i1.10139

Bonn, S., Alezandrou, C., Hjörleifsdottir, K., Wiklander, K., Östenson, C.G., Löf, M., \& Trolle, Y. (2018). App-technology to increase physical activity among patients with diabetes type 2 - the DiaCertstudy, a randomized controlled trial. BMC Public Health, 18(119), 17. DOI:10.1186/s12889-018-5026-4

Casanova, A., \& Yuste, M.J. (2016). Efecto y modo de aplicación de la educación terapéutica en las enfermedades respiratorias crónicas: revisión sistemática. Cuestiones de fisioterapia, 45(2), 120-135.

Castro, N., \& Gómez, I. (2016). Incorporación de los códigos QR en la Educación Física en Secundaria. Retos, 29, 114-119.

Chacón-Borrego, F., Ubago, J.L., La Guardia, J.J., Padial, R., \& Cepero, M. (2018). Educación e higiene postural en el ámbito de la Educación Física. Papel del maestro en la prevención de lesiones. Revisión sistemática. Retos, 34, 8-13.

Conde, M., \& Tercedor, P. (2015). La actividad física, la educación física y la condición física pueden estar relacionadas con el rendimiento académico y cognitivo en jóvenes. Revisión sistemática. Archivos de medicina del deporte, 32(2), 100-109.

Conroy, D.E., Yang, C.H., \& Maher, J. (2014). Behavior Change Techniques in Top-Ranked Mobile Apps for Physical Activity. American Journal of Preventive Medicine, 46(6), 649-652. DOI:10.1016/j.amepre.2014.01.010

Cuadri, J., Tornero, I., Sierra, A., \& Sáez, J.M. (2018). Revisión sistemática sobre los estudios de intervención de actividad física para el tratamiento de la obesidad. Retos, 33, 261-266.

Díaz, J. (2018). Mejorar el aprendizaje en acción integrando mobile learning en la educación física. Tándem: Didáctica de la Educación Física, 60, 69-73.

Direito, A., Jiang, Y., Whittaker, R., \& Maddison, R. (2015). Smartphone apps to improve fitness and increase physical activity among young people: protocol of the Apps for Improving FITness (AIMFIT) randomized controlled trial. BMC Public Health, 15(635), 1-12. DOI:10.1186/s12889-015-1968-y

Fanning, J., Mullen, S.P., \& McAuley, E. (2012). Increasing Physical Activity With Mobile Devices:A Meta-Analysis. Journal of medical internet research, 14(6), 1-11. DOI:10.2196/jmir.2171

Filgueira, J.M. (2014). Mobile-Learning: aplicaciones educativas en Android para el profesor de Educación Física. Revista de Educación Física, 30(2), 1-10.

Filgueira, J.M. (2016). Apps Para Adquisición de Hábitos Saludables Dentro de la Educación Física. Revista de Educación Física, 34(4), 2231.

Fukuoka, Y., Lindgren, T., Dov, Y., Hooper, J., \& Aswani, A. (2018). Applying Natural Language Processing to Understand Motivational Profiles for Maintaining Physical Activity After a Mobile App and Accelerometer-Based Intervention: The mPED Randomized Controlled Trial. JMIR Mhealth Uhealth, 6(6), 1-11. DOI:10.2196/ 10042

Gabbiadini, A., \& Greitemeyer, T. (2018). Fitness mobile apps positively affect attitudes, perceived behavioural control and physical activities. Journal of Sports Medicine and Physical Fitness, 4, 1-21. DOI:10.23736/S0022-4707.18.08260-9

Gallego, V., Muñoz, J.A., Arribas, H.F., \& Rubia, B. (2016). Aprendizaje ubicuo: un proceso formativo en educación física en el medio natural. 
RELATEC, 15(1), 59-73. DOI:10.17398/1695288X.15.1.59

García, N., \& Sánchez, S. (2014). Incorporación de dispositivos móviles a la Educación Física escolar. Revista Española de Educación Física y Deportes, 407, 79-86.

García-Umaña, A., \& Tirado, R. (2018). Digital Media Behavior of School Students: Abusive Use of the Internet. NAER: Journal of New Approaches in Educational Research, 7(2), 140-147. DOI:10.7821/ naer.2018.7.284

Glynn, L., Hayes, P., Casey, M., Glynn, F., Alvarez-Iglesias, A., Newell, J., Ólaighin, G., Heaney, D., \& Murphy, A. (2013). SMART MOVE - a smartphone-based intervention to promote physical activity in primary care: study protocol for a randomized controlled trial. Trials, 14(157), 1-7. DOI:10.1186/1745-6215-14-157

Gómez, M., Trujillo, J.M., Aznar, I., \& Cáceres, M.P. (2018). Augment reality and virtual reality for the improvement of spatial competences in Physical Education. Journal of Human Sport and Exercise, 2, 189-198. DOI:10.14198/jhse.2018.13.Proc2.03

Greco, G., Tambolini, R., Ambruosi, P., \& Fischetti, F. (2017). Negative effects of smartphone use on physical and technical performance of young footballers. Journal of Physical Education and Sport, 17(4), 2495-2501. DOI:10.7752/jpes.2017.04280

Guillen, L., Herrera, A.P., \& Ale, Y. (2018). Las herramientas tecnológicas TIC's como elemento alternativa para el desarrollo del componente físico. Retos, 34, 222-229.

Höchsmann, C., Walz, S., Schäfer, J., Holopainen, J., Hanssen, H., \& Schmidt, A. (2017). Mobile Exergaming for Health-Effects of a serious game application for smartphones on physical activity and exercise adherence in type 2 diabetes mellitus - study protocol for a randomized controlled trial. Trials, 18(103), 1-17. DOI:10.1186/ s13063-017-1853-3

Howe, K.B., Suharlim, C., Ueda, P., Howe, D., Kawachi, I., \& Rimm, E.B. (2016). Gotta catch'em all! Pokémon GO and physical activity among young adults: difference in differences study. BMJ, 355, 1-4 DOI:10.1136/bmj.i6270

Leirós, R., Arce, M.E., García-Soidán, J., \& Naveira, G. (2017) Accelerometers: Devices that contribute to healthy aging. Retos, 32, 44-47.

Lozano, A.M., Zurita, F., Ubago, J.L., Puertas, P., Ramírez, I., \& Núñez, J.I. (2019). Videojuegos, práctica de actividad física, obesidad y hábitos sedentarios en escolares de entre 10 y 12 años de la provincia de Granada. Retos, 35, 42-46.

Marcelo, C., Yot, C., Álvarez, J.A., Ortega, J.A., \& Aros, A. (2016) Learning in mobility with Context4Learning: Developing a contextaware mobile learning application. International Journal of Mobile Learning and Organisation, 10(4), 203-222. DOI:10.1504/ IJMLO.2016.079497

Marqués, L., Cela, J., \& Gisbert, M. (2017). Pre-service Physical Education Teachers' self-management ability: a training experience in 3D simulation environments. Retos, 32, 30-34.

Martine, J., Mennes, M., Alpay, L., Bijwaard, H., \& Baart, M. (2015) App use, physical activity and healthy lifestyle: a cross sectional study. BMC Public Health, 15(883), 1-9. DOI:10.1186/s12889-0152165-8

Martínez-López, E.J., Moreno, J., Suarez, S., \& Ruiz-Ariza, A. (2018) Efecto y satisfacción de un programa de actividad física controlada por pulsómetro en el índice de masa corporal de escolares con sobrepeso-obesidad. Retos, 33, 179-184.

Mascarenhas, M., Mayllin, J., Vittinghoff, E., Lynn, E., \& Hecht, F. (2018). Increasing Physical Activity in Mothers Using Video Exercise Groups and Exercise Mobile Apps: Randomized Controlled Trial. Journal of Medical Internet Research, 20(5), 1-14. DOI:10.2196/ jmir.9310

Monguillot, M., González, C., Guitert, M., \& Zurita, C. (2014). Mobile learning: una experiencia colaborativa mediante códigos QR. Revista de Universidad y Sociedad del Conocimiento (RUSC), 11(1), 175191. DOI:10.7238/rusc.v11i1.1899

Moreno, R., Fernández, A.B., Linares, M., \& Espejo, T. (2018). Revisión sistemática sobre hábitos de actividad física en estudiantes universitarios. Sportis, 4(1), 162-183. DOI:10.17979/sportis.2018.4.1.206

Muntaner, A., Vidal-Conti, J., \& Palou, P. (2016). Increasing physical activity through mobile device interventions: A systematic review. Health Informatics Journal, 22(3), 451-469. DOI:10.1177/ 1460458214567004

Nagovitsyn, R.S., Vladykina, I.V., \& Senator, S. (2015). Training program to hit standards of all-Russian sports complex-ready for labour and
defence-(GTO) based on mobile learning. Teoriya i Praktika Fizicheskoy Kultury, 1, 46-48.

Núñez, J.I., Zurita, F., Ramírez, I., Lozano, A.M., Puertas, P., \& Ubago, J.L. (2019). Análisis de la relación entre los hábitos físico-saludables y la dieta con la obesidad en escolares de tercer ciclo de Primaria de la Provincia de Granada. Retos, 35, 31-35.

Pérez-López, I.J., Rivera, E., \& Trigueros, C. (2017). «La profecía de los elegidos»: un ejemplo de gamificación aplicado a la docencia universitaria. Revista Internacional de Medicina y Ciencias de la Actividad Física y el Deporte, 17(66), 243-260. DOI:10.15366/ rimcafd2017.66.003

Prat, Q., Camerino, O., \& Coiduras, J.L. (2013). Introducción de las TIC en educación física. Estudio descriptivo sobre la situación actual. Apunts. Educación Física y Deportes, 113(3), 37-44. DOI:10.5672/ apunts.2014-0983.es.(2013/3).113.03

Quintero, L.E., Jiménez, F., \& Area, M. (2018). Claves para la integración y el uso didáctico de los dispositivos móviles en las clases de Educación Física. AcciónMotriz, 20, 17-26.

Rodríguez-García, A.M., Romero, J.M., \& Agreda, M. (2019). Impact of ICT on the teaching of Physical Education: a bibliometric research study. ESHPA - Education, Sport, Health and Physical Activity, 3(1), 1-14. DOI:http://hdl.handle.net/10481/53211

Safran, J., Madar, Z., \& Shahar, D. (2015). The Impact of a Web-Based App (eBalance) in Promoting Healthy Lifestyles: Randomized Controlled Trial. Journal of Medical Internet Research, 17(3), 1-14. DOI:10.2196/jmir.3682

Sánchez-Meca, J. (2003). La revisión del estado de la cuestión: el metaanálisis. En C. Camisón, M.J. Oltra y M.L. Flor (Eds.), Enfoques, problemas y métodos de investigación en Economía y Dirección de Empresas. Tomo I (pp. 101-110). Castellón: Universitat Jaume I.

Soler, F.B., Sánchez-Meca, J., López, J.M., \& Navarro, F. (2014). Neuroticismo y trastorno por estrés postraumático: un estudio metaanalítico. Revista Española de Salud Pública, 88, 17-36. DOI:10.4321/S1135-57272014000100003

Steinberg, D., Levine, E., Askew, S., Foley, P., \& Bennett, G. (2013). Daily Text Messaging for Weight Control Among Racial and Ethnic Minority Women: Randomized Controlled Pilot Study. Journal of Medical Internet Research, 15(11), 1-11. DOI:10.2196/jmir.2844

Suárez, C., Lloret, C., \& Mengual, S. (2016). Percepción docente sobre la transformación digital del aula a través de tabletas: un estudio en el contexto español. Comunicar, 24(49), 81-89. DOI:10.3916/C492016-08

Turner-McGrievy, G., Wilcox, S., Boutté, A., Hutto, B., Singletary, C., Muth, E., \& Hoover, A.W. (2017). The Dietary Intervention to Enhance Tracking with Mobile Devices (DIET Mobile) Study: A 6Month Randomized Weight Loss Trial. Obesity, 25, 1336-1342. DOI:10.1002/oby.21889

Urrútia, G., \& Bonfill, X. (2010). Declaración PRISMA: una propuesta para mejorar la publicación de revisiones sistemáticas y metaanálisis. Medicina Clínica, 135(11), 507-511. DOI:10.1016/ j.medcli.2010.01.015

Valentiner, L.S., Ried-Larsen, M., Karstoft, K., Fau, C., Brøns, C., Oestergaard, R., Christense, R., Steen, J., Arthur, A., Klarlund, B., \& Langberg, H. (2017). Long-term effect of smartphone-delivered Interval Walking Training on physical activity in patients with type 2 diabetes: protocol for a parallel group single-blinded randomised controlled trial. BMJ Open, 7, 1-16. DOI:10.1136/bmjopen-2016014036

Van Dantzig, S., Geleijnse, G., \& Van Halteren, A.T. (2013). Toward a persuasive mobile application to reduce sedentary behaviour. Personal and Ubiquitous Computing, 17, 1237-1246. DOI:10.1007/ s00779-012-0588-0

Willcox, J.C., Wilkinson, S.A., Lappas, M., Ball, K., Crawford, D., McCarthy, E.A., Fjeldsoe, B., Whittaker, R., Maddison, R., \& Campbell, K.J. (2017). A mobile health intervention promoting healthy gestational weight gain for women entering pregnancy at a high body mass index: the txt4two pilot randomised controlled trial. BJOG. An International Journal of Obstetrics \& Gynaecology, 124, 1718-1728. DOI:10.1111/1471-0528.14552

Yang, H.J., Kang, J.H., Hyun, O., Choi, M., Oh, M., Nam, J., \& Sung, E. (2017). Interventions for Preventing Childhood Obesity with Smartphones and Wearable Device: A Protocol for a Non-Randomized Controlled Trial. International Journal of Environmental Research and Public Health, 14(184), 1-10. DOI:10.3390/ijerph14020184 